\title{
ESPECIES DEPREDADORAS DE TRIPS (THYSANOPTERA) ASOCIADAS A HUERTAS DE AGUACATE EN NAYARIT, MÉXICO
}

\author{
Jhonathan Cambero-Campos, ${ }^{1}$ Roberto Johansen-Naime, ${ }^{2}$ Oswaldo \\ García-Martínez, ${ }^{1}$ Mario Cantu-Sifuentes, ${ }^{3}$ Ernesto Cerna-Chavez ${ }^{1}$ y Axel \\ Retana-Salazar 5 \\ ${ }^{1}$ Departamento de Parasitología Agrícola, Universidad Autónoma Agraria Antonio Narro, Buenavista, \\ Saltillo, Coahuila, México, C.P. 25315. Jhony695@gmail.com \\ ${ }^{2}$ Colección Nacional de Insectos, Instituto de Biología, Universidad Nacional Autónoma de México. \\ A.P. 70-153. C.P. 04510. México, D.F. \\ ${ }^{3}$ Departamento de Estadística, UAAAN, Buenavista, Saltillo, Coahuila, México, C.P. 25315. \\ ${ }^{4}$ Centro de Investigación en Estructuras Microscópicas (CIEMic), Ciudad de la Investigación, \\ Universidad de Costa Rica 2060, San José, Costa Rica.
}

Cambero-Campos, J., R. Johansen-Naime, O. García-Martínez, M. Cantu-Sifuentes, E. CernaChavez \& A. Retana-Salazar. 2011. Especies depredadoras de trips (Thysanoptera) asociadas a huertas de aguacate en Nayarit, México. Acta Zool. Mex. (n. s.), 27(1): 115-121.

RESUMEN. El objetivo fue identificar las especies de trips depredadoras asociadas al cultivo del aguacate en el Estado de Nayarit. El trabajo se llevó a cabo en cuatro huertas de aguacate del cultivar "Hass" y una con los cultivares "Choquette" y "Hall". Se realizaron muestreos semanales de enero a diciembre de 2008, utilizando las técnicas de muestreo de derribo y redeo. Se determinaron 294 trips con hábitos depredadores, resultando 16 especies. Franklinothrips vespiformis fue la especie más representada (21.8 $\%$, seguida de Leptothrips primigenus (21.4\%), y F. orizabensis (19.0\%), las restantes especies recolectadas representaron el $37.8 \%$.

Palabras clave: Hass, Choquette, Hall, Aeolothrips, Erythrothrips, Franklinothrips, Scolothrips, Leptothrips, Trybomia.

Cambero-Campos, J., R. Johansen-Naime, O. García-Martínez, M. Cantu-Sifuentes, E. CernaChavez \& A. Retana-Salazar. 2011. Predator species of thrips (Thysanoptera) associated with avocado orchards in Nayarit, Mexico. Acta Zool. Mex. (n. s.), 27(1): 115-121.

ABSTRACT. The objective of this study was to identify the predaceous thrips species associated with the avocado crop in the state of Nayarit. This research was carried out in four avocado orchards of the cultivars Hass, Choquette and Hall. Weekly samples were taken from January to December, 2008, using the knockdown and net sweeping sampling methods. A total of 294 thrips were identified as predators within 16 species. Franklinothrips vespiformis was the dominant species $(21.8 \%)$ followed by Lepto-

Recibido: 05/05/2008; aceptado: 06/08/2010. 
thrips primigenus $(21.4 \%)$ and $F$. orizabensis $(19.0 \%)$, the remaining species collected accounted for $37.8 \%$.

Keys words: Hass, Choquette, Hall, Aeolothrips, Erythrothrips, Franklinothrips, Scolothrips, Leptothrips, Trybomia.

\section{INTRODUCCIÓN}

México es el principal productor de aguacate a nivel mundial, con una producción de $1,124,565$ ton, cosechadas en 114,471 ha (FAOSTAT 2008). En el país, el aguacate se cultiva en 26 Estados; siendo Nayarit el segundo lugar nacional en cuanto a volumen de producción anual y superficie establecida, con alrededor de 2703 ha (SIAP 2009). El cultivo presenta varias especies de ácaros e insectos fitófagos que afectan la calidad y cantidad de producción, considerándose a los trips como una de las principales plagas, debido a que causan lesiones en hojas, flores y frutos pequeños cuando se alimentan (González-Hernández et al. 2000). Para el control de estos organismos, el método más usado es el control químico (Dughetti 1990), lo que ha creado problemas como la resistencia y el impacto ambiental por su mal uso. Es por ello que existen estudios que buscan otras alternativas como es el uso de depredadores (Lacasa \& Llorens 1998).

Desde hace tiempo se conoce la presencia de trips depredadores en los cultivos, a pesar de no haber sido reconocidos debido a que otras especies de este grupo presentan gran reputación como plaga (Kirk 1997). La acción depredadora de los trips tiene como función principal satisfacer sus necesidades nutricionales y en segundo lugar corresponden a las relacionadas con la competencia por espacio en la planta o en un órgano de esta (Lacasa \& Llorens 1998). Sabelis \& Van Rinj (1997) enlistan 23 especies de Thysanoptera como depredadoras efectivas. En el Neotrópico existe poca información de estos enemigos naturales (Mound \& Marullo 1996). En México se conocen 10 especies depredadoras en agroecosistemas de aguacate (Johansen-Naime et al. 2007), pero ninguna de estas ha sido registrada para el Estado de Nayarit.

El objetivo del presente trabajo fue identificar las especies de Thysanoptera depredadoras con potencial para ser evaluadas en programas de control biológico, recolectadas mediante diferentes técnicas de muestreo (redeo y derribo) en la región aguacatera del Estado de Nayarit.

\section{MATERIAL Y MÉTODOS}

Para la obtención de trips, se llevaron a cabo muestreos semanales de enero a diciembre del 2008, en cinco huertas de aguacate, localizadas en los tres municipios (Tepic, Xalisco, San Blas) de la región aguacatera de Nayarit. En Tepic se recolectó en dos huertas del cultivar Hass, la primera en el Ejido Camichin de Jauja denominada Tintilagua $\left(21^{\circ} 29^{\prime} 18.5^{\prime \prime} \mathrm{N}, 1^{\circ} 4^{\circ} 46^{\prime} 18.4^{\prime \prime} \mathrm{W}, 1117 \mathrm{msnm}\right)$, siendo la única huerta 
con manejo orgánico, y la segunda en El Fortín (21³1’00.5” N, 10458’57.8” W, $1,099 \mathrm{msnm})$ del Ejido Venustiano Carranza. En Xalisco dos huertas del cultivar Hass, la huerta Chapula $\left(21^{\circ} 25^{\prime} 09.4^{\prime \prime} \mathrm{N}, 104^{\circ} 54^{\prime} 53.9^{\prime}\right.$ ' W, $\left.1,035 \mathrm{msnm}\right)$ dentro del

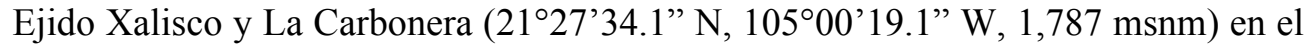
Ejido el Cuarenteño. En San Blas se consideró la huerta El Cedro (21 $32^{\circ}$ '47.4" N, $105^{\circ} 08^{\prime} 23.1$ ” W, $384 \mathrm{msnm}$ ) del Ejido Mecatan con los cultivares Coquette y Hall. Las últimas cuatro huertas con manejo convencional.

Para la obtención de trips, se utilizaron el derribo y el redeo como técnicas de muestreo de acuerdo a Cambero-Campos et al. (2009), esto con la finalidad de recolectar tanto a los trips presentes en el dosel del árbol como a los de la maleza de las huertas. Los insectos recolectados se colocaron en frascos con alcohol etílico al 70\%. El material biológico recolectado se trasladado al Laboratorio de Entomología del Departamento de Parasitología Agrícola (DPA) de la Universidad Autónoma Agraria Antonio Narro (UAAAN) para su montaje e identificación. En laboratorio, se separaron los trips adultos en buen estado del resto de los insectos, para luego montarlos de acuerdo a la técnica desarrollada por Johansen-Naime \& Mojica-Guzmán (1997). La determinación de las especies, se realizó con el apoyo de literatura y utilizando claves taxonómicas (Johansen-Naime 1983; Johansen-Naime 1987; Mound \& Marullo 1996; Soto-Rodríguez \& Retana-Salazar 2003). Los números de captura entre huertas y tipo de captura, se compararon a través de la prueba estadistica Ji-cuadrada de Pearson (Pearson 1900). Los especímenes identificados fueron depositados en la Colección Nacional de Insectos de la Universidad Autónoma de México, en el Centro de Investigación en Estructuras Microscópicas (CIEMic), Universidad de Costa Rica y en la Colección de Insectos del Departamento de Parasitología Agrícola, UAAAN, Buenavista, Saltillo, Coahuila, México,

\section{RESULTADOS}

En el Cuadro 1 se aprecia que durante los 12 meses de muestreo, en las cinco huertas, con las dos técnicas de muestreo, se capturaron un total de 9428 trips (diferentes estadios). Con la técnica de derribo se capturó el mayor número (8558) equivalente al $90.7 \%$, mientras que con el redeo solo 870 individuos (9.3\%) (Cuadro 1); estas proporciones resultaron ser significativamente diferentes $\left(\mathrm{X}^{2}=6269.129, \mathrm{gl}=1, p\right.$ $<<0.001)$.

En la huerta La Carbonera se obtuvo el mayor número de capturas (41.5\%), seguida por El Cedro (19.3\%), Tintilagua (17.9\%), El Fortín (11.8\%) y Chapula (9.6\%) respectivamente. Estos datos resultaron ser significativamente diferentes $\left(\mathrm{X}^{2}=\right.$ 3028.267, $\mathrm{gl}=4, p<<0.001)$.

Del total de trips recolectados, se montaron e identificaron los adultos en buen estado (980), equivalente al 10.4\% del total capturados, resultando de estos 294 indi- 
Cuadro 1. Total de trips recolectados por técnica de muestreo y por huerta de aguacate del estado de Nayarit, México.

\begin{tabular}{|c|c|c|c|c|c|}
\hline \multirow[b]{2}{*}{ Municipio } & \multirow[b]{2}{*}{ Huerta } & \multicolumn{2}{|c|}{ Capturas } & \multirow[b]{2}{*}{ Total } & \multirow[b]{2}{*}{$\%$} \\
\hline & & Redeo & Derribo & & \\
\hline \multirow[t]{2}{*}{ Xalisco } & Chapula & 173 & 728 & 901 & 9.6 \\
\hline & Carbonera & 152 & 3758 & 3910 & 41.5 \\
\hline \multirow[t]{2}{*}{ Tepic } & El Fortín & 133 & 979 & 1112 & 11.8 \\
\hline & Tintilagua & 220 & 1467 & 1687 & 17.9 \\
\hline San Blas & El Cedro & 192 & 1626 & 1818 & 19.3 \\
\hline Total & & 870 & 8558 & 9428 & 100 \\
\hline
\end{tabular}

viduos con hábitos depredadores (30\%) (Cuadro 2), de los cuales 278 se capturaron mediante el derribo $(94.5 \%)$ y 16 por redeo $(5.5 \%)$. El mayor número de capturas se registró en La Carbonera con 81 individuos y el menor en El Cedro (36) (Cuadro 2); esta última, la única huerta con los cultivares Choquette y Hall quedando intermedia Tintilagua con 59, (huerta orgánica). En el Cuadro 2 se muestran las 16 especies identificadas, de las cuales siete corresponden a la familia Aeolothripidae, una a Thripidae y ocho a Phlaeothripidae. Franklinothrips vespiformis fue la especie más representada (21.8\%), seguida de Leptothrips primigenus (21.4\%), y F. orizabensis (19.04\%). El resto de las especies tuvieron porcentajes en ámbitos de 0.34 a 14.62 .

En los árboles de las huertas muestreadas, se detectó la presencia de grandes cantidades de ácaros, de los cuales es posible se alimenta Scolothrips sexmaculatus y las especies de Leptothrips. Se detectó la presencia de Trybomia brevitubus en bajos porcentajes. Cabe destacar la presencia de cuatro especies nuevas, una perteneciente a Franklinothrips y tres a Leptothrips.

\section{DISCUSIÓN}

Estos resultados son similares a los publicados por Cambero-Campos et al. (2009), quienes mencionan la técnica del derribo, como la mejor forma de capturar trips. Mencionan Cambero-Campos et al. (2007) a la Carbonera como la huerta que presentó el mayor número de capturas $(40.5 \%)$, estos datos pudieran deberse a que esta huerta se ubica a $1787 \mathrm{msnm}$, mientras que las huertas restantes se localizan por debajo de los $1400 \mathrm{msnm}$ y es posible que los vientos a esa altitud propician la riqueza de especies según lo observado por Mound \& Marullo (1996).

Las familias encontradas en este trabajo ya habían sido registradas para el aguacate Hass de Nayarit por Úrias-López et al. (2007) y Cambero-Campos et al. (2007). Estos últimos autores mencionan a Aeolothripidae, como la más representada con $71.5 \%$, esta familia está conformada por 190 especies, la gran mayoría depredadoras de ácaros y pequeños artrópodos (Hoddle et al. 2008), aunque existen algunas 
Cuadro 2. Especies de trips depredadores recolectados en cinco huertas de aguacate de los municipios de Xalisco, Tepic y San Blas, Nayarit.

\begin{tabular}{|c|c|c|c|c|c|c|c|}
\hline \multirow[b]{2}{*}{ Especies } & \multicolumn{5}{|c|}{ Número de especímenes } & \multirow[b]{2}{*}{ Total } & \multirow[b]{2}{*}{$\%$} \\
\hline & Cha & Car & For & Tin & Ced & & \\
\hline Aeolothrips mexicanus Priesner, $1924^{\text {A }}$ & 5 & 3 & 4 & 2 & - & 14 & 4.8 \\
\hline Erythrothrips durango Watson, $1924^{\mathrm{A}}$ & 9 & 12 & 9 & 9 & 4 & 43 & 14.6 \\
\hline Franklinothrips lineatus Hood, $1949^{\text {A }}$ & - & 6 & 4 & 1 & - & 11 & 3.7 \\
\hline Franklinothrips orizabensis Johansen, $1974^{\text {A }}$ & 6 & 20 & 20 & 10 & - & 56 & 19.0 \\
\hline Franklinothrips vespiformis (D.L. Crawford, 1909) ${ }^{\text {A }}$ & 8 & 6 & 12 & 17 & 21 & 64 & 22.0 \\
\hline Franklinothrips caballeroi Johansen, $1980^{\mathrm{A}}$ & - & - & - & 1 & - & 1 & 0.3 \\
\hline Franklinothrips sp. nov ${ }^{\mathrm{A}}$ & 1 & - & 1 & 2 & 2 & 6 & 2.0 \\
\hline Scolothrips sexmaculatus (Pergande, 1894) ${ }^{\mathrm{T}}$ & 4 & 1 & 1 & 4 & - & 10 & 3.4 \\
\hline Leptothrips astutus Johansen, $1978^{\mathrm{P}}$ & - & - & 1 & - & - & 1 & 0.3 \\
\hline Leptothrips longicapitis Johansen, $1987^{\mathrm{P}}$ & - & 5 & 2 & 1 & - & 8 & 2.7 \\
\hline Leptothrips obesus Johansen, $1987^{\mathrm{P}}$ & 1 & 1 & - & - & - & 2 & 0.7 \\
\hline Leptothrips primigenus Johansen, $1987^{\mathrm{P}}$ & 15 & 25 & 11 & 9 & 3 & 63 & 21.4 \\
\hline Leptothrips sp. nov. $2^{\mathrm{P}}$ & 1 & 1 & 1 & - & - & 3 & 1.0 \\
\hline Leptothrips sp. nov. $3^{\mathrm{P}}$ & - & - & - & 1 & 1 & 2 & 0.7 \\
\hline Leptothrips sp. nov. $4^{\mathrm{P}}$ & 2 & 1 & - & - & - & 3 & 1.0 \\
\hline Trybomia brevitubus (Moulton, 1929) ${ }^{\mathrm{P}}$ & - & - & - & 2 & 5 & 7 & 2.4 \\
\hline Total & 52 & 81 & 66 & 59 & 36 & 294 & 100 \\
\hline
\end{tabular}

Cha-Chapula, Car-Carbonera, For-Fortín, Tin-Tintilagua, Ced-Cedro.

${ }^{\mathrm{A}}$ Aeolothripidae, ${ }^{\mathrm{T}}$ Thripidae, ${ }^{\mathrm{P}}$ Phlaeothripidae.

especies que se alimentan de polen (Mound \& Marullo 1996). Estos autores también mencionan que en las familias Thripidae y Phlaeothripidae existen algunas especies con hábitos depredadores.

Mound \& Reynaud (2005), proveen una clave para las 14 especies de Franklinothrips reconocidas a nivel mundial. En este género, tanto los adultos como las larvas son depredadores obligados de pequeños artrópodos y pueden ser observados activamente buscando a su presa (Hoddle 2003a, b). Varios miembros de este género, son considerados agentes de control biológico exitosos contra trips de interés fitosanitario. Algunas especies parecen ser arbóreas, viven en las hojas de árboles posiblemente en las partes altas del dosel, otras ocurren en arbustos, mientras que $F$. vespiformis es comúnmente encontrada a ras de suelo en hierbas pequeñas (Loomans \& Heijboer 1999; Loomans \& Vierbergen 1999).

Hoddle et al. (2008), mencionan que $S$. sexmaculatus se encuentra particularmente en árboles frutales y se considera depredador de ácaros al igual que las especies de 
Leptothrips, género muy complejo considerado de Haplothrips por Stannard (1957). Johansen-Naime (1987) realizó la revisión taxonómica de 41 especies de Leptothrips en el Continente Americano, de la cuales 22 fueron nuevas, de las que se incluyen en este trabajo: L. astutus, L. obesus, L. primigenus y L. longicapitis. La principal dificultad que se presenta para estudiar la biología de las especies de Leptothrips, se deriva de la escasez de individuos inmaduros en estado natural.

En cuanto a Trybomia brevitubus existe poca información sobre su actividad depredadora, mencionada por Johansen-Naime et al. (2007) como visitadora activa de agallas de Trioza anceps (Tuthill) en follaje de aguacate mexicano.

Estos nuevos hallazgos, enriquecen la información de la fauna mexicana de Thysanoptera, e indican que aún hay regiones con gran cantidad de especies nuevas que podrían tener importancia en el desarrollo de controles biológicos, como es el caso de estas cuatro especies, las cuales deben estudiarse más a fondo en su biología y ecología. Lo anterior justifica la necesidad de incentivar en regiones como Nayarit los estudios de biodiversidad y ecología de estos insectos, tanto por contener especies plaga como depredadoras con potencial en implementar programas de control biológico. Los estudios sobre trips en los estados de la costa del Pacífico no son abundantes por lo que es necesario no sólo determinar su variabilidad y diversidad, sino los factores ambientales (agroclimáticos) y prácticas agrícolas que afectan su distribución y riqueza como lo han demostrado Mound \& Marullo (1996) para Centroamérica.

AGRADECIMIENTOS. Los autores agradecen al personal del Laboratorio de Parasitología de la Universidad Autónoma de Nayarit su valioso apoyo para la realización de este trabajo. Así como a los dos árbitros anónimos que revisaron y mejoraron el manuscrito.

\section{LITERATURA CITADA}

Cambero-Campos, O. J., R. Johansen-Naime, O, García-Martínez, C. Carvajal-Cazola, N. Isiordia-Aquino, \& M. Cantú-Sifuentes. 2009. Thrips fitófagos en huertas de aguacate cv. Hass, en Nayarit, México. Brenesia, 71-72: 61-64.

Cambero-Campos, O. J., R. Johansen-Naime, M. Soto-Hernandez, N. Isiordia-Aquino, O. OrtegaAvalos, \& M. Cantú-Sifuentes. 2007. Thysanoptera Asociada a Huertos de Aguacate cv. Hass en el Municipio de Xalisco Nayarit, México. In: Entomología Mexicana. Memorias del Congreso de Entomología, 6: 1355-1359.

Dughetti, A. C. 1990. Evolución de insecticidas en el control de trips de la cebolla. EEA.INTA Hilario Ascasubi. Informe Técnico. No. 34, 10 p.

FAOSTAT. 2008. Database results. (En línea). Consultada 13 de Marzo 2010. Disponible en: http:// faostat.fao.org/site/567/DesktopDefault.aspx?PageID=567\#ancor.

González-Hernández, H., R. Johansen-Naime, C. L. Gasca, A. Equihua-Martínez, A. Salinas, E. Estrada, F. Durán, \& A. Valle. 2000. Plagas del aguacate pp: 117-121. In: Daniel Téliz (ed). El Aguacate y su Manejo Integrado. Editorial Mundi Prensa México, S. A. de C. V. México.

Hoddle, M. S. 2003a. Predation behaviours of Franklinothrips orizabensis (Thysanoptera: Aeolothripidae) towards Scirtothrips perseae and Heliothrips haemorroidalis (Thysanoptera: Thripidae). Biological Control, 27: 323-328. 
Hoddle, M. S. 2003b .The effect of prey species and environmental complexity on the functional response of Franklinothrips orizabensis: a test of the fractal foraging model. Ecological Entomology, 28: 309-318.

Hoddle, M. S., L. A. Mound, \& D. Paris. 2008. Thrips of California. CBIT Publishing, Queensland. (En línea). Consultada 20 de febrero 2010. Disponible en: http://keys.lucidcentral.org/keys/v3/ thrips_of_california/Thrips_of_California.html

Johansen-Naime, R. M. 1983. Nuevos estudios acerca del mimetismo en el género Franklinothrips Back (Insecta: Thysanoptera) en México, Anales del Instituto de Biología UNAM. Serie Zoología, 53: 133-156.

Johansen-Naime, R. M. 1987. El género Leptothrips Hood, 1909 (Thysanoptera: Phlaeothripidae) en el Continente Americano. Su sistemática, filogenia, biogeografía, biología, conducta y ecología. Monografías del Instituto de Biología. UNAM,.3: 1-246.

Johansen-Naime, R. M., A. Mojica-Guzmán, H. González-Hernández, A. R. Valle de la Paz, E. L. Castañeda-González, G. Ávila-Quezada, \& C. M. Sosa-Torres. 2007. Trips asociados con el aguacate en México. pp. 134-153. In: Téliz, M. y Mora, A. (Eds). El aguacate y su manejo integrado. Mundiprensa. México.

Johansen-Naime, R. M. \& A. Mojica-Guzmán. 1997. Importancia agrícola de los thrips. In: Manual sobre entomología y acarología aplicada del 22 al 24 de mayo. UPAEP, Puebla, Pue. SME-UPAEP. pp. 11-18.

Kirk, W. D. J. 1997. Feeding. pp: 119-174. In: T. Lewis (Ed.). Thrips as a Crop Pest. CAB International, Wallingford, Oxon.

Lacasa, A. \& J. M. Llorens. 1998. Trips y su control biológico (II). Pisa Ed., Alicante, pp 312.

Loomans, A. J. M. \& G. Vierbergen. 1999. Franklinothrips: perspectives for greenhouse pest control. Bulletin IOBC/WPRS, 22: 157-190.

Loomans, A. J. M. \& A. Heijboer. 1999. Franklinothrips vespiformis (Crawford): a polyphagous predator preying on thrips eggs. Proceedings of the section Experimental and Applied Entomology of the Netherlands Entomological Society (N.E.V.), 10: 143-150.

Mound, L. A. \& R. Marullo. 1996. The thrips of central and south America: an introduction (Insecta; Thysanoptera). Memoirs on Entomology International. 6: 1-487.

Mound, L. A. \& P. Reynaud. 2005. Franklinothrips; a pantropical Thysanoptera genus of ant-mimicking obligate predators (Aeolothripidae). Zootaxa, 864: 1-16.

Pearson, K. 1900. On the criterion that a given system of deviations from the probable in the case of a correlated system of variables is such that it can be reasonably supposed to have arisen from random sampling. Philosophical Magazine, 50: 157-175.

Sabelis, M. W. \& P. C. J. Van Rijn. 1997. Predation by Insects and Mites. pp: 259-354. In: T. Lewis (Ed.). Thrips as a Crop Pest. CAB International, Wallingford, Oxon.

SIAP. 2009. (En línea). Consultada 20 de Abril 2010. Disponible en: http://reportes.siap.gob.mx/Agricola siap/ResumenProducto.do?producto $=600 \&$ invitado $=$ true $\&$ ciclo $=3$

Soto-Rodríguez. G. A. \& A. P. Retana-Salazar. 2003. Clave ilustrada para los géneros de Thysanoptera y especies de Frankliniella presentes en cuatro zonas hortícolas en Alajuela, Costa Rica. Agronomía Costarricense, 27: 55-68.

Stannard, L. J. 1957. The phylogeny and classification of the North American genera of the sub-order Tubulifera (Thysanoptera). Illinois Biological Monographs, 25: 1-200.

Úrias-López, M. A., S. Salazar-García, R. Johansen-Naime. 2007. Identificación y fluctuación poblacional de especies de trips (Thysanoptera) en aguacate 'hass' en Nayarit, México. Rev. Chapingo. Serie horticultura, 13: 49-54. 